\title{
Fractional generalization of memristor and higher order elements
}

\author{
J. Tenreiro Machado
}

\begin{abstract}
A B S T R A C T
Fractional calculus generalizes integer order derivatives and integrals. Memristor systems generalize the notion of electrical elements. Both concepts were shown to model important classes of phenomena. This paper goes a step further by embedding both tools in a generalization considering complex-order objects. Two complex operators leading to real-valued results are proposed. The proposed class of models generate a broad universe of elements. Several combinations of values are tested and the corresponding dynamical behavior is analyzed.
\end{abstract}

Keywords:

Fractional calculus

Memristor

Device modeling

\section{Introduction}

In the competing intellectual paradigms proposed by science, history reveals that, besides the common steady progress, quantum leaps occur, often being recognized as milestones by future generations [1,2].

In 1695 the speculations of Gottfried Leibniz about a derivative of order $\frac{1}{2}$ lead to a new branch of the integral and differential calculus usually known as 'fractional calculus' (FC). This term entails the birth of the idea, but is actually a misnomer and the designation 'integration and differentiation of arbitrary order' is more appropriate [3,4]. FC remained an abstract mathematical speculation until 1893, when Oliver Heaviside applied this tool in the areas of operational calculus and electromagnetism [5-7]. However, only in the last decades researchers verified that FC models capture intrinsically many characteristics of natural and artificial phenomena, while the integer order counterpart lacks describing easily those properties.

In 1953 Fermi, Pasta and Ulam developed computer simulations of a system composed of masses and nonlinear springs [8]. The nonlinear terms demonstrated a new rich gallery of dynamical behaviors not seen previously with linear models. Their numerical experiments marked the beginning of the nonlinear physics and the era of computer analysis of scientific problems [9-11]. Nonlinear dynamics is an unquestionable paradigm of present day scientific research and sheds light in all areas of science.

In 1971 Chua [12] noticed possible symmetries in electrical elements and variables. Somehow inspired by the periodic table of elements discovered by Dmitri Mendeleev and Julius Meyer in the 19th century, Chua speculated that besides the well known resistor, capacitor and inductor, a forth element, that he denoted 'memristor', would be necessary to complete the diagram. Thirty-seven years later this abstract conceptualization was proved to have real world applications [13] and more ambitious generalizations are now being formulated.

These examples of scientific debate demonstrate that generalization of concepts and broader horizons are an important process in human knowledge. Bearing these ideas in mind, this paper studies the generalization of the memristor in the perspective of fractional order systems, and is organized as follows. Section two introduces the main concepts in mathematics and engineering. Sections three develops numerical experiments with nonlinear fractional systems. Finally, section four outlines the main conclusions. 


\section{Preliminary concepts}

This section introduces the three main concepts adopted in this paper. Section 2.1 presents the fundamentals of fractional calculus (FC). Section 2.2 describes the developments of the ideas underlying the memristor. Section 2.3 discusses the implementation of the frequency-dependent negative resistor (FDNR).

\subsection{Fractional calculus}

FC generalizes the concept of differentiation $\frac{d^{p} f(x)}{d x^{p}}$ to non-integer, or even complex, orders $p[14,15]$.

FC was born in 1695 from speculations of Gottfried Leibniz. In a letter to Guillaume l'Hôpital, Leibniz raised the question 'Can the meaning of derivatives with integer order be generalized to derivatives with non-integer orders?' l'Hôpital replied posing another question to Leibniz: 'What if the order will be $\frac{1}{2}$ ?' Leibniz replied 'It will lead to a paradox, from which one day useful consequences will be drawn'. In 1738 Euler noticed that the evaluation of $\frac{d^{p} x^{a}}{d x^{p}}$ had meaning for non-integer values $p$. Later, in 1812, Laplace studied the differentiation of non-integer order for certain classes of functions. FC has been an ongoing topic for 300 years and many important mathematicians paid attention to the topic over the years, among them Fourier, Abel, Liouville, Riemann, and Weyl. Historical surveys can be found in the books by Oldham and Spanier [16], Miller and Ross [17], Samko and Marichev [18].

FC was developed merely in the scope of pure mathematics and only by 1893, with the pioneer work of Heaviside [19] related with electromagnetism and operational calculus, applications in physics and engineering emerged. In 1936 the application of FC in the area of viscoelasticity was also addressed by Gemant [20,21]. We can mention also Blair [22,23] that in 1944 applied FC in rheology. Unfortunately, the work of these visionaries, ahead of their time, was forgotten during some decades. The first conference in FC is due to Ross in 1974 [24] who organized the First Conference on fractional calculus and its Applications at the University of New Haven. The last two decades brought considerable attention to FC, stimulated by its findings in many areas of applied sciences [25-29]. We can find applications of FC in control theory, chemical physics, stochastic processes, anomalous diffusion, rheology, and many other [30,31,14,32-36].

There are several different definitions of fractional derivatives and their comparison is outside the scope of this subsection. The most used definitions of a fractional derivative of order $\alpha$ are, respectively, the Riemann-Liouville, Grünwald-Letnikov and Caputo formulations:

$$
\begin{aligned}
& \left.{ }_{a}^{R L} D_{t}^{\alpha} f(t)=\frac{1}{\Gamma(n-\alpha)} \frac{d^{n}}{d t^{n}} \int_{a}^{t} \frac{f(\tau)}{(t-\tau)^{\alpha-n+1}} d \tau, t>a, \operatorname{Re}(\alpha) \in\right] n-1, n[ \\
& { }_{a}^{G L} D_{t}^{\alpha} f(t)=\lim _{h \rightarrow 0} \frac{1}{h^{\alpha}} \sum_{k=0}^{\left[\frac{t-a]}{h}\right]}(-1)^{k}\left(\begin{array}{l}
\alpha \\
k
\end{array}\right) f(t-k h), \quad t>a, \alpha>0 \\
& { }_{a}^{C} D_{t}^{\alpha} f(t)=\frac{1}{\Gamma(n-\alpha)} \int_{a}^{t} \frac{f^{(n)}(\tau)}{(t-\tau)^{\alpha-n+1}} d \tau, \quad t>a, n-1<\alpha<n
\end{aligned}
$$

where $\Gamma()$ is Euler's gamma function, $[x]$ means the integer part of $x$, and $h$ is the step time increment.

One important property of these operators is that they capture the history of all past events contrary to what occurs with integer derivatives that are 'local' operators. This means that fractional order systems have intrinsically a memory of the previous dynamical evolution. This behavior has been recognized in several natural and man made phenomena and their modeling becomes much simpler using the tools of FC, while the counterpart of building integer order models leads often to complicated expressions. The geometrical interpretation of fractional derivatives has been the subject of debate and several perspectives had been forwarded [37-40].

Using the Laplace transform we have the expressions:

$$
\begin{aligned}
& \mathcal{L}\left\{{ }_{0}^{R L} D_{t}^{\alpha} f(t)\right\}=s^{\alpha} \mathcal{L}\{f(t)\}-\sum_{k=0}^{n-1} s^{k}{ }_{0}{ }^{R L} D_{t}^{\alpha-k-1} f\left(0^{+}\right) \\
& \mathcal{L}\left\{{ }_{0}{ }^{C} D_{t}^{\alpha} f(t)\right\}=s^{\alpha} \mathcal{L}\{f(t)\}-\sum_{k=0}^{n-1} s^{\alpha-k-1} f^{(k)}(0)
\end{aligned}
$$

where $s$ and $\mathcal{L}$ denote the Laplace variable and operator, respectively.

The Mittag-Leffler function $E_{\alpha}(x)$ is defined as [41]:

$$
E_{\alpha}(x)=\sum_{k=0}^{\infty} \frac{x^{k}}{\Gamma(\alpha k+1)}, \quad \alpha \in C, \operatorname{Re}(\alpha)>0, x \in C
$$


The Mittag-Leffler function forms a bridge between the exponential and the power laws. The first occurs in phenomena governed by integer order and the second in fractional order dynamics. In particular, when $\alpha=1$ Mittag-Leffler function simplifies and we have $E_{1}(x)=e^{x}$, while, for large values of $x$, the asymptotic behavior yields:

$$
E_{\alpha}(-x) \approx \frac{1}{\Gamma(1-\alpha)} \frac{1}{x}, \quad \alpha \neq 1,0<\alpha<2
$$

Since the Laplace transform leads to:

$$
\mathcal{L}\left\{E_{\alpha}\left( \pm a t^{\alpha}\right)\right\}=\frac{s^{\alpha-1}}{S^{\alpha} \mp a}
$$

we observe a generalization of the Laplace transform pairs from the exponential towards the Mittag-Leffler, namely from integer up to fractional powers of $s$.

A typical application of FC is in the area of control [42] and corresponds to the generalization of the Proportional, Integral and Derivative (PID) algorithm, namely to the fractional PID, denoted $P I^{\lambda} D^{\mu}$ by Podlubny [43]. The diagram of Fig. 1 shows that the cases $(\lambda, \mu)=\{(0,0),(1,0),(0,1),(1,1)\}$, correspond to the $P, P I, P D$ and $P I D$, respectively.

During the last years efforts were devoted to implement the fractional impedance, called by some as 'fractor'. The idea comes from the models of the three fundamental electrical elements, constituted by the inductor $L$, resistor $R$, and capacitor C:

$$
\begin{aligned}
& v(t)=L \frac{d i(t)}{d t} \\
& v(t)=\operatorname{Ri}(t) \\
& v(t)=\frac{1}{C} \int_{-\infty}^{t} i(\tau) d \tau
\end{aligned}
$$

where $v(t)$ and $i(t)$ represent the electrical voltage and current, respectively, and $t$ denotes time. Obviously, analogies with other types of systems can be easily established. For example, in a mechanical system we have the mass, the viscous damping and the spring. The important thing is that the three elements represent integer order implementations and we guess that intermediary (fractional) cases may exist. In the Laplace representation a fractional capacitor or a fractional inductor of order $0<\alpha<1$ have impedances $Z(s)=\frac{1}{s^{\alpha} C}$ and $Z(s)=s^{\alpha} L$, respectively. Research on this topic proved the existence of fractional capacitors and inductors [44-53].

The conjunction of fractional and nonlinear dynamics has been also addressed in the last years and we can mention several studies [54-58].

\subsection{Memristor and higher-order elements}

In 1971 Chua [12] noticed the symmetry between electrical elements and variables depicted in Fig. 2, where $v(t)$ and $i(t)$ represent the electrical voltage and current, and $t$ denotes time. The magnetic flux and the electrical charge, $\phi$ and $q$, are related to the previous variables by means of the expressions:

$$
\phi(t)=\int_{-\infty}^{t} v(\tau) d \tau
$$

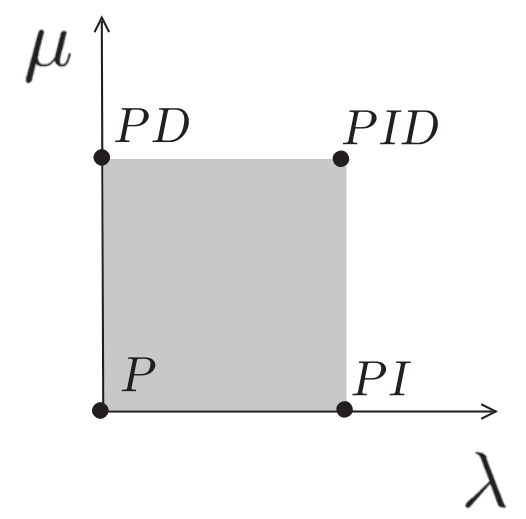

Fig. 1. The $P I^{\lambda} D^{\mu}$ and the four integer cases $P, P I, P D$ and $P I D$. 


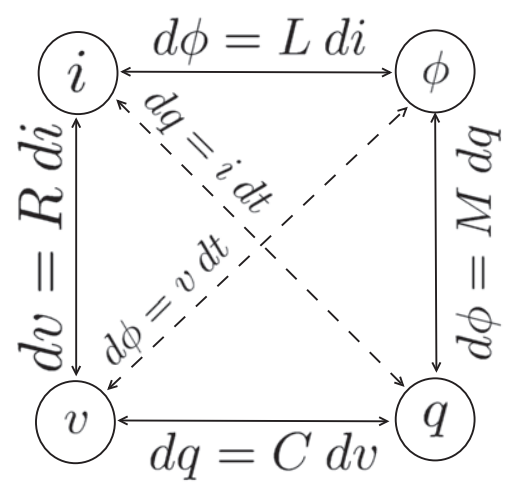

Fig. 2. The four fundamental one-port circuit elements: resistor, capacitor, inductor and memristor [59,13].

$$
q(t)=\int_{-\infty}^{t} i(\tau) d \tau
$$

In classical linear circuit theory the three fundamental elements are constituted by the resistor $R$, inductor $L$ and capacitor $C$, modeled as:

$$
\begin{aligned}
& v(t)=R i(t) \\
& \phi(t)=L i(t) \\
& q(t)=C v(t)
\end{aligned}
$$

Chua proposed that there should be a fourth basic element $M$, which he called 'memristor', for memory resistor, completing the set of relations with:

$$
\phi(t)=M q(t)
$$

In the case of a linear relationship, $M$ is a constant and is similar to a resistance, but if $M$ is a function of $q$, then we have a nonlinear element. Oster and Auslander [60] analyzed the concept using Bond graphs and applied it to tapered dashpot and to a electrolytic tank. In 1976 Kang and Chua [61] generalized the memristor concept to a larger class of nonlinear systems, called as 'memristive systems'. Later, in 1980, 2003 and 2009 Chua et al. [59,62,63] developed these ideas and formulated a periodic table of circuit elements. These conjectures were mainly abstract, but in 2008 the scientific community was awaken by a paper of Stewart et al. [13]. Researchers in HP laboratories [64] were studying small electrical devices and proposed a cross bar structure with a thin layer of titanium dioxide sandwiched between two platinum contacts. Classical modeling requires a time-varying and frequency-dependent resistance [65], while the adoption of the memristor concept leads to a neat and concise description of all phenomena. Researchers realized that other scientific areas should pay attention to the 'new' concept for modeling natural phenomena [66,67]. Recently Chua et al. [68] noticed that, in fact, the distinction between 'memristors' and 'memristive systems' is not significant and recommended the adoption of a simplified nomenclature, namely the original memristor. Doria-Cerezo and Jeltsema [69] are addressing memristive ports in the perspective of Hamiltonian systems.

The charge-controlled memristor is modeled by the expression:

$$
\phi=\hat{\phi}(q)
$$

Calculating the time derivative it yields:

$$
v=\frac{\partial \hat{\phi}(q)}{\partial q} i
$$

where $M_{i}(q)=\frac{\partial \hat{\phi}(q)}{\partial q}$ (units in $\Omega$ similarly to a resistance) is the incremental memresistence.

The dual case consists of the flux-controlled memristor and is modeled by the expressions:

$$
\begin{aligned}
& q=\hat{q}(\phi) \\
& i=\frac{\partial \hat{q}(\phi)}{\partial \phi} v
\end{aligned}
$$

where $M_{v}(\phi)=\frac{\partial \hat{q}(\phi)}{\partial \phi}$ (units in $\Omega^{-1}$ similarly to a conductance) is the incremental memconductance. 
Expressions (19) and (21) establish the relationship $v=0 \Longleftrightarrow i=0$. Furthermore, when (18) (or (20)) is linear we obtain the standard linear resistance $R$ (conductance $G$ ).

The one-port higher-order element proposed by Chua represented in Fig. 3 establishes a relation between $v(t)$ and $i(t)$ of the type:

$$
\frac{d^{n}}{d t^{n}} u(t)=f\left(\frac{d^{m}}{d t^{m}} i(t)\right), \quad n, m=\ldots-2,-1,0,1,2, \ldots
$$

Clearly the cases $(n, m)=\{(0,0),(-1,0),(0,-1),(-1,-1)\}$, correspond to a resistor, inductor, capacitor and memresistor, respectively.

Based on this concept, the periodic table of all two-terminal circuit elements [63] is represented in Fig. 4.

As pointed Doria-Cerezo and Jeltsema [70] if we have:

$$
\begin{aligned}
& \sigma(t)=\int_{-\infty}^{t} \nu(\tau) d \tau \\
& \rho(t)=\int_{-\infty}^{t} \phi(\tau) d \tau
\end{aligned}
$$

then, similarly to what we found in (17) for the memristor, relationships can be defined between charge, flux, integrated charge, and integrated flux:

$$
\begin{aligned}
& \sigma=\hat{\sigma}(\phi) \\
& \rho=\hat{\rho}(q) \\
& q=C_{M}(\phi) v \\
& \phi=L_{M}(q) i
\end{aligned}
$$

where $C_{M}(\phi)=\frac{\partial \hat{\sigma}(\phi)}{\partial \phi}$ and $L_{M}(q)=\frac{\partial \hat{\rho}(q)}{\partial q}$ (same units as capacitance and inductance) are the incremental memcapacitor and meminductor. As for the memresistor, the memory aspect of the flux-modulated memcapacitor, or the charge-modulated meminductor, stems from the fact that they 'remember' the amount of voltage, or of current, that were applied to them. In [70] Jeltsema and Dòria-Cerezo assign the symbol "?" for the relation between $\phi$ and $\sigma$. One possibility is to denote it as memristor of second order and to write $d \sigma=M_{M} d \rho$, but that notation remains to be defined. The results are summarized in Fig. 5.

When the memristor $M_{i}$ is linear, Eq. (19) reduces to the Ohms law. However, when the relationship is nonlinear things become more interesting. Stewart et al. [13] conceived a physical model of the HP device where the magnetic flux $\phi$ is a quadratic function of the charge $q$, and the nonlinear relationship between the integrals of the current and voltage for a memristor is achieved.

Let us consider a two-terminal $M_{i}(q)$ charge-controlled memristor connected to a current source $i(t)=\sin \left(\omega_{0} t\right)$ such that $\phi(t)=\frac{1}{3} q^{3}(t)$. Fig. 6 depicts the $v(q)$ versus $i(q)$ for $\omega_{0}=\{1,5\}$. The plot is two-valued, with a hysteresis pinched loop, with exception of the origin where $v=0 \Longleftrightarrow i=0$ as mentioned previously. For higher values of $\omega_{0}$ we observe that the characteristic tends to a straight line. From the practical point of view there are difficulties in defining this element based on the measurements of $v$ and $i$ which point that a model defined by $\phi$ and $q$ is more appropriate. Furthermore, the relevance of the concept is associated with the presence of a nonlinear system and its memory effect upon the global dynamics.

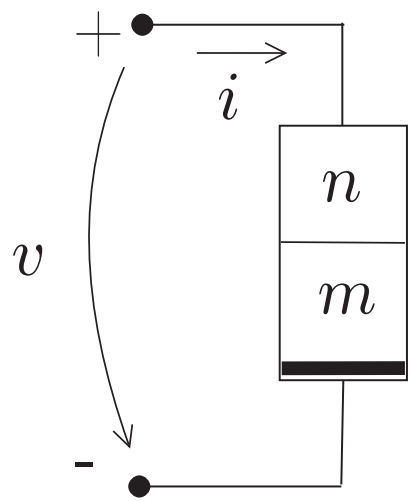

Fig. 3. One-port higher-order element. 


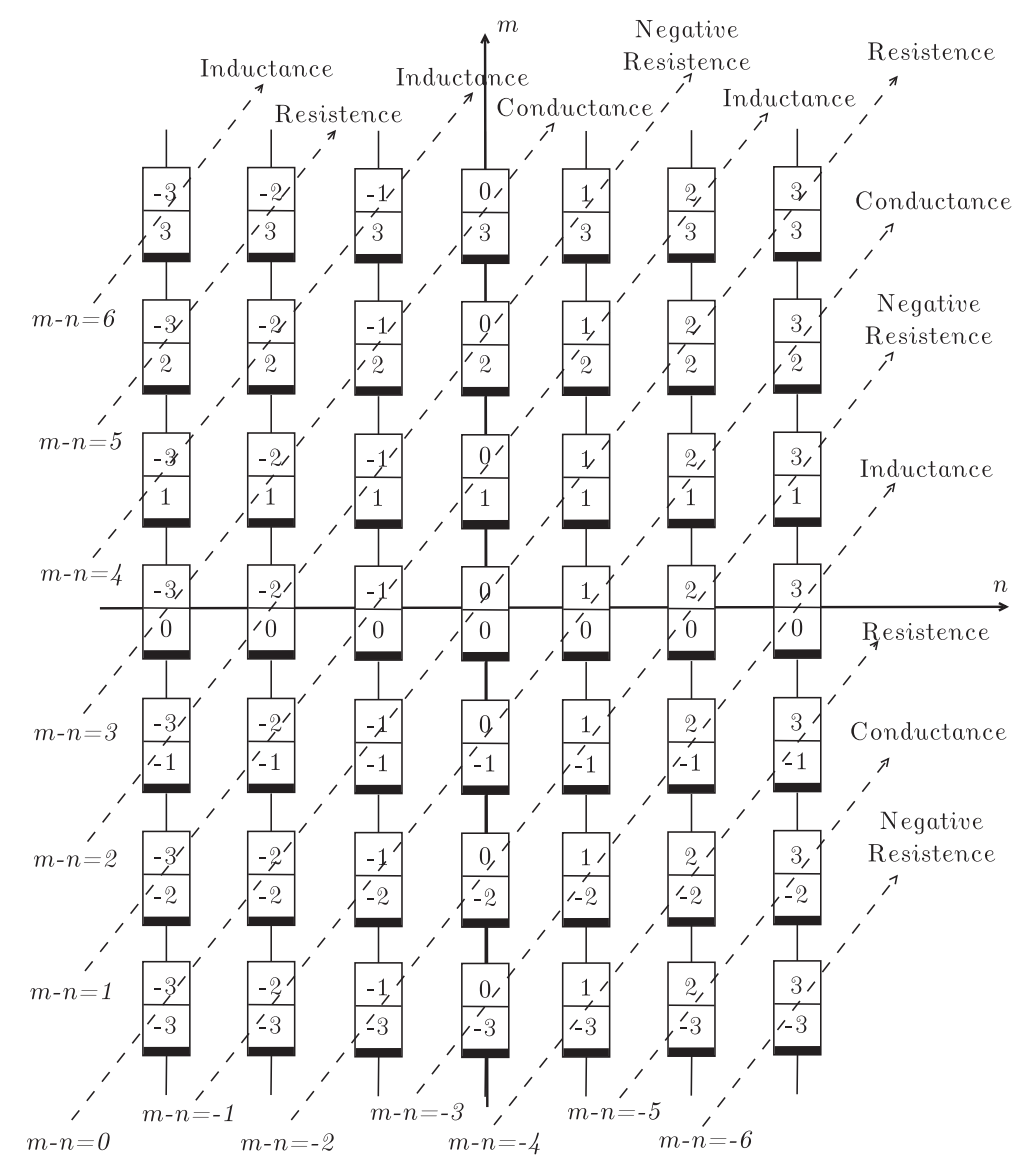

Fig. 4. Periodic table of all two-terminal circuit elements $[59,62]$.

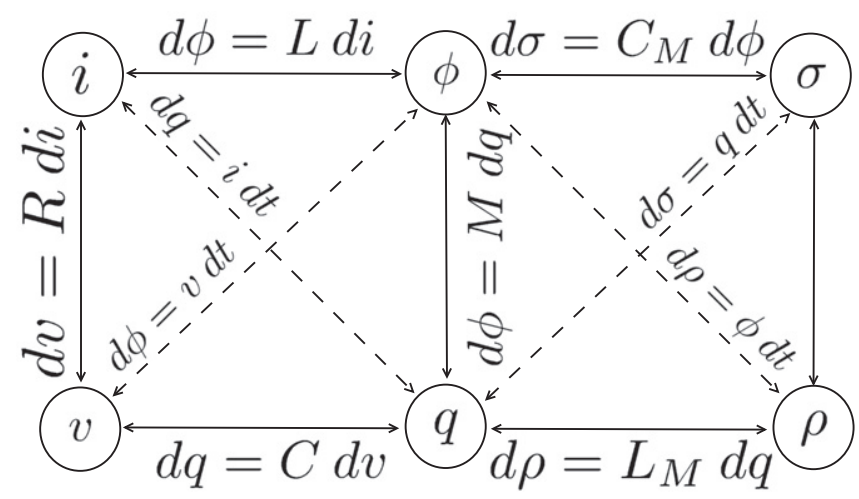

Fig. 5. Charge-controlled memristor $M_{i}(q): v(q)$ versus $i(q)$ for $\omega_{0}=\{1,5\}$ [70].

\subsection{Frequency dependent negative resistances}

The FDNR was introduced in 1969 by Bruton [71] for the realization of ladder filters. In 1970 Antoniou [72] developed several realizations of the generalized impedance converter (CGIC). The circuit adopts the operational amplifier as the active element for the synthesis of voltage transfer functions. The current conveyor (CCII) [73] was used in realizing grounded and floating inductors and FDNR elements. The first realization of an ideal grounded FDNR was reported in [74] and this topic has been under progress during the last decades $[75,76]$.

Fig. 7 shows the block diagram of a CGIC $[77,78]$. In the Laplace domain the input impedance $Z(s)$ of this circuit is: 


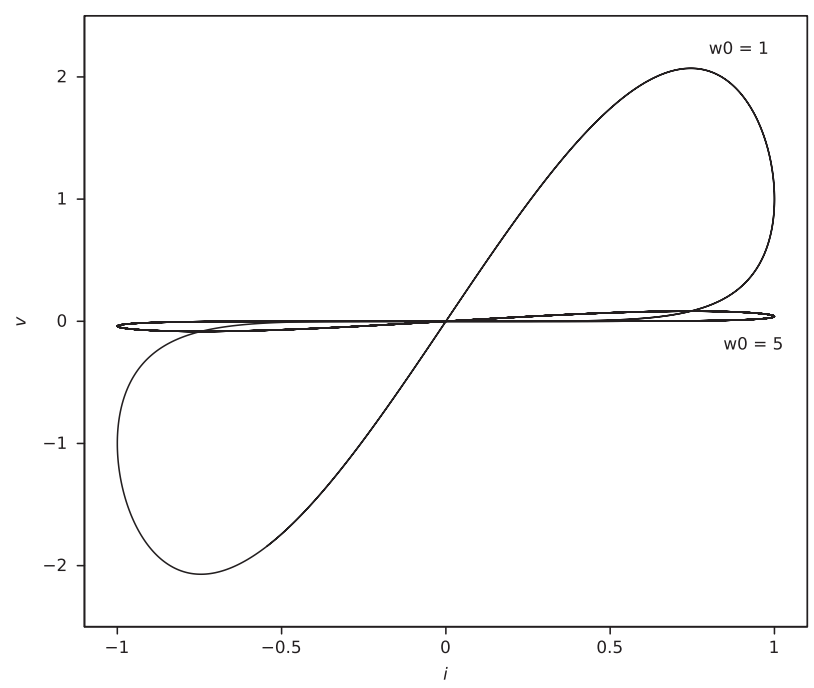

Fig. 6. Charge-controlled memristor $M_{i}(q): v(q)$ versus $i(q)$ for $\omega_{0}=\{1,5\}$.

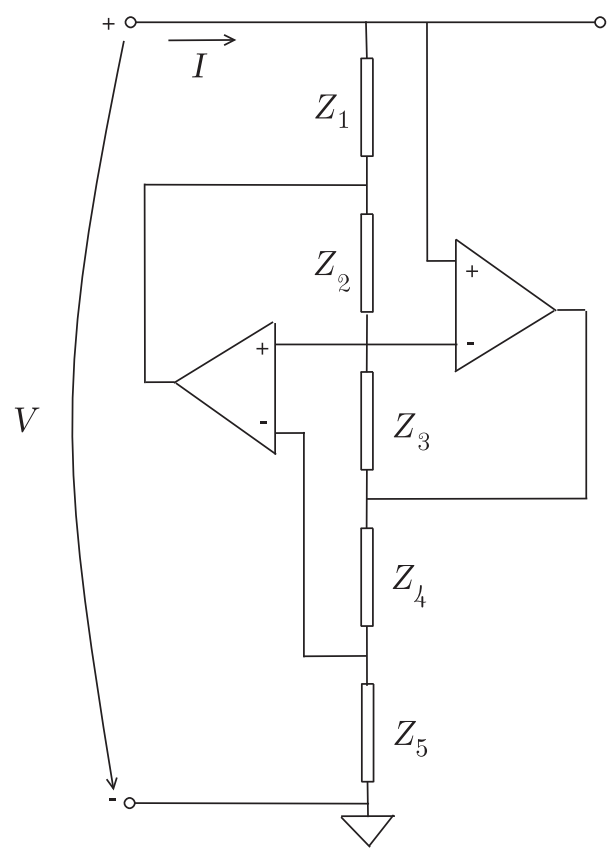

Fig. 7. Generalized impedance converter.

$$
Z(s)=\frac{V(s)}{I(s)}=\frac{Z_{1}(s) Z_{3}(s) Z_{5}(s)}{Z_{2}(s) Z_{4}(s)}
$$

where $Z_{i}(s), i=\{1, \ldots, 5\}$, denote the impedance of each branch of the circuit.

By substituting some impedances by capacitors in appropriate locations and having the rest as resistors, several impedances can be synthesized, namely floating inductors and FDNR elements. For example, having two capacitors and three resistors with impedances $Z_{1}(s)=\frac{1}{s C_{1}}, R_{2}, Z_{3}(s)=\frac{1}{s C_{3}}, R_{4}, R_{5}$, results the FDNR impedance:

$$
Z(s)=\frac{R_{5}}{s^{2} C_{1} C_{3} R_{2} R_{4}}
$$

This impedance, which is often called a $D$ element, has the value $D=C_{1} C_{3} R_{2} R_{4} R_{5}^{-1}$. 
FDNR have been used mainly in ladder filters without inductors, where the impedance of each two-terminal element is multiplied by a factor $\frac{1}{s}$. This means that an inductor, resistor and capacitor, with impedances $Z(s)=s L, Z(s)=R$ and $Z(s)=\frac{1}{s C}$ are transformed into a resistor, capacitor and FDNR, with impedances $Z(s)=L, Z(s)=\frac{R}{s}$ and $Z(s)=\frac{1}{s^{2} C}$, respectively.

Two main aspects should be retained. The first is that the FDNR requires the implementation by means of active devices, but demonstrates that higher order elements, although not passive, are physically possible. The second is that while FDNR are being used in electronics for linear applications there is no theoretical limitation for their application in nonlinear systems.

It is worth noticing an aspect that has not been explored. For $Z_{i}(s)$ of integer order we get a circuit input impedance $Z(s)$ also of integer order. However, combining impedances of fractional order, let us say $Z_{i}(s)=k_{i} s^{\alpha_{i}}$, we can obtain $Z(s)$ of integer and fractional order, both capacitive and inductive, not limited to values of \pm 1 .

\section{Generalization of memresitors and fractances}

It was observed recently [79-81] that the concepts underlying the fractional impedances and the meristor could be joined, but some deeper analysis is required. In fact, we can redraw the fundamental relationships of Fig. 2 as depicted in Fig. 8 and then in subdividing the first order integral $I^{1}$ as two fractional integrals $I^{1-\alpha}$ and $I^{\alpha}$ represented in Fig. 9. The elements that involve energy storage/dissipation are indicated by the diagonal/vertical harrows. This means that the elements resistor and memristor have now a fractional order counterpart between the variables $I^{\alpha} i(t)$ and $I^{\alpha} v(t)$.

Fig. 10 shows the $v-i$ plane for $i(t)=\sin \left(\omega_{0} t\right), \phi=\frac{1}{3} q^{3}, \omega_{0}=1$, and $I^{\alpha} v=M_{i}(q) I^{\alpha} i, \alpha=\left\{\frac{1}{4}, \frac{1}{2}, \frac{3}{4}, 1\right\}$. We verify that as $\alpha$ approaches one we get a chart similar to the one obtained in the integer case. However, we have now distinct shapes in the positive and negative 'leafs' of the pinched loop. The energy added to/removed from the system is the area between the curve and the axis. In all cases the areas of the 'leafs' are identical and each leaf gives the amount of added/removed energy in one half-period.

This embedding of the two concepts is straightforward from Sections 2.1 and 2.2, but some two other aspects can be added. A first argument comes from the Chua periodic table of elements presented in 2.2 and the FDNR discussed in 2.3 . We verify that we have a 'continuum' and the orders can theoretically come from $-\infty$ up to $+\infty$, although it is still to be demonstrated if all elements are physically possible. The second argument comes from recent incursions in the area of complex order derivatives [82-88].

For a sine function, at steady-state, the complex derivative of order $\alpha \pm j \beta$ is given by:

$$
D^{\alpha \pm \imath \beta} \sin (\omega t)=\omega^{\alpha} \cdot\{\cos [\beta \ln (\omega)] \pm j \sin [\beta \ln (\omega)]\} \cdot\left[\sin \left(\omega t+\alpha \frac{\pi}{2}\right) \cosh \left(\beta \frac{\pi}{2}\right) \pm j \cos \left(\omega t+\alpha \frac{\pi}{2}\right) \sinh \left(\beta \frac{\pi}{2}\right)\right]
$$

where $j=\sqrt{-1}$ and $\omega$ is the angular frequency.

For obtaining real-valued results, we can group the conjugate orders, into the operators:

$$
\begin{aligned}
& \mathrm{CO}_{1}^{(\alpha, \beta)} \sin (\omega t)=\frac{1}{2}\left[D^{\alpha+j \beta}+D^{\alpha-j \beta}\right] \sin (\omega t) \\
& \mathrm{CO}_{1}^{(\alpha, \beta)}=\omega^{\alpha}\left[\cos [\beta \ln (\omega)] \sin \left(\omega t+\alpha \frac{\pi}{2}\right) \cosh \left(\beta \frac{\pi}{2}\right)-\sin [\beta \ln (\omega)] \cos \left(\omega t+\alpha \frac{\pi}{2}\right) \sinh \left(\beta \frac{\pi}{2}\right)\right] \\
& \mathrm{CO}_{2}^{(\alpha, \beta)} \sin (\omega t)=\frac{1}{2 j}\left[D^{\alpha+\imath \beta}-D^{\alpha-\imath \beta}\right] \sin (\omega t) \\
& \mathrm{CO}_{2}^{(\alpha, \beta)} \sin (\omega t)=\omega^{\alpha}\left[\cos [\beta \ln (\omega)] \cos \left(\omega t+\alpha \frac{\pi}{2}\right) \sinh \left(\beta \frac{\pi}{2}\right)+\sin [\beta \ln (\omega)] \sin \left(\omega t+\alpha \frac{\pi}{2}\right) \cosh \left(\beta \frac{\pi}{2}\right)\right]
\end{aligned}
$$

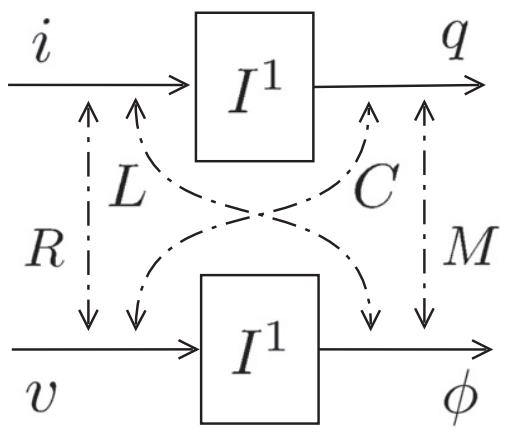

Fig. 8. The four fundamental integer order circuit elements: resistor, capacitor, inductor and memristor. 


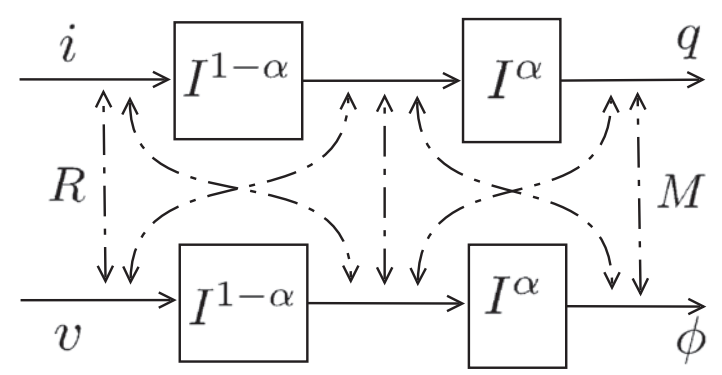

Fig. 9. Fractional order circuit elements.

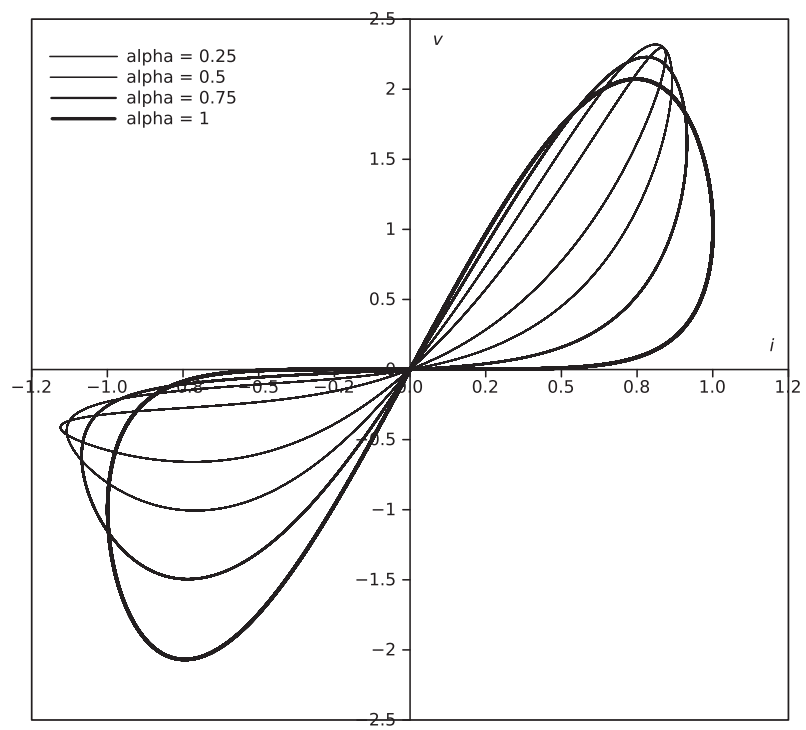

Fig. 10. Charge-controlled memristor $I^{\alpha} v=M_{i}(q) I^{\alpha} i: v(q)$ versus $i(q)$ when $\omega_{0}=1$ and $\phi=\frac{1}{3} q^{3}, \alpha=\left\{\frac{1}{4}, \frac{1}{2}, \frac{3}{4}, 1\right\}$.

We have now the operators $\mathrm{CO}_{1}^{(\alpha, \beta)}$ and $\mathrm{CO}_{2}^{(\alpha, \beta)}$ that reduce to $D^{\alpha}$ and 0 , respectively, when $\beta=0$. Each operator has two parameters and their combination leads to a rich field of possibilities. Let us recall the example presented in Section 2.2 of the two-terminal $M_{i}(q)$ charge-controlled memristor connected to a current source $i(t)=\sin \left(\omega_{0} t\right)$, $\omega_{0}=1$, such that $\phi(t)=\frac{1}{3} q^{3}(t)$. For implementing the operators was considered a numerical approach in the Fourier domain, as follows:

1. the input signal $i(t)$ is integrated leading to the charge $q(t)$,

2. the non-linear dynamics $\phi=\frac{1}{3} q^{3}$ produces the flux $\phi(t)$

3. the Fourier transform is applied to $q(t)$ and $\phi(t)$,

4. the complex order operator $C O_{1}^{(\alpha, \beta)}\left(\right.$ or $\left.C O_{2}^{(\alpha, \beta)}\right)$ is applied by means of the multiplication with $\frac{1}{2}\left[(j \omega)^{\alpha+j \beta}+(j \omega)^{\alpha-j \beta}\right]($ or $\left.\frac{1}{2 j}\left[(j \omega)^{\alpha+j \beta}-(j \omega)^{\alpha-j \beta}\right]\right)$,

5. the time evolution of the signals is recovered by means of the inverse Fourier transform.

Since a indetermination occurs for $\omega=0$ the bias point of the memristor operating was centered numerically. In all cases the areas of the two leafs remained similar although their shapes varied significantly.

Fig. 11 depicts the results for the $\mathrm{CO}_{1}$ and $\mathrm{CO}_{2}$, when $\omega_{0}=1$ and $(\alpha, \beta)=\left(\frac{1}{2}, \frac{3}{2}\right)$.

For analysing the influence of the pair $(\alpha, \beta)$ upon the results was decided to examine the area $A$ of one leaf of the pinched loop for several combinations of values. Fig. 12 shows the locus of $A$ versus $(\alpha, \beta)$ for the $C O_{1}^{(\alpha, \beta)}$ and $C O_{2}^{(\alpha, \beta)}$, respectively.

We conclude that there is a kind of 'periodic behavior' with $\alpha$, with period 2, and that $\beta$ has a considerable influence (near a logarithmic relationship) upon the magnitude of the excursions. Furthermore, $\mathrm{CO}_{1}^{(\alpha, \beta)}$ and $\mathrm{CO}_{2}^{(\alpha, \beta)}$ lead to distinct results, but the overall dynamics, in terms of the variation of the area $S$ with $(\alpha, \beta)$, has some resemblances.

It remains to be seen whether this generalization is merely an abstract speculation, or if the elements of this complex order universe are physically possible. Nevertheless, there is already the proposal for including complex order operators in control algorithms [89]. On the other hand, the influence of the memory build in the nonlinear dynamics and the memory intrinsic to FC may also to be further explored. 


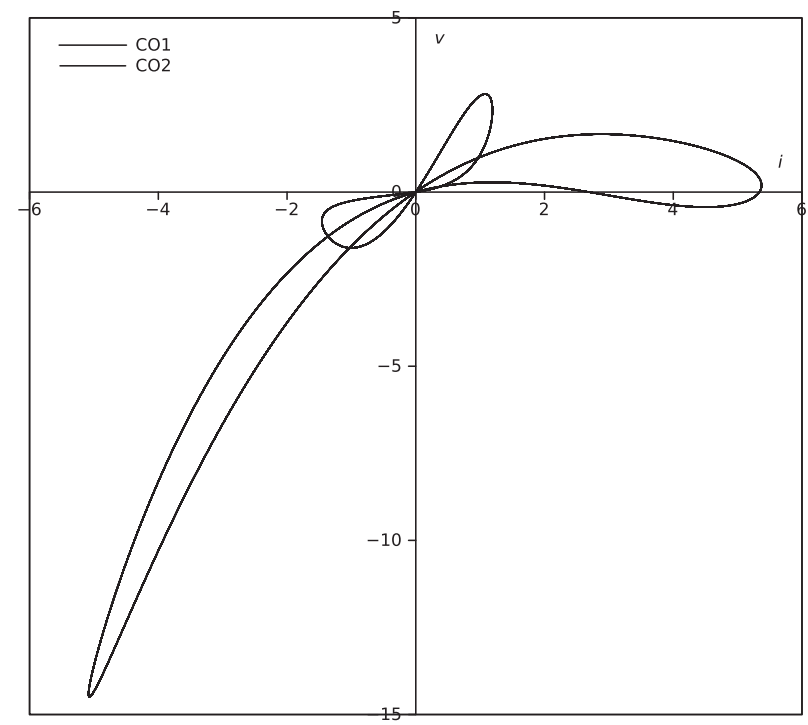

Fig. 11. Charge-controlled memristor $M_{i}(q): v(q)$ versus $i(q)$ for the $C O_{1}^{(\alpha, \beta)}$ and $C O_{2}^{(\alpha, \beta)}$, when $\omega_{0}=1,(\alpha, \beta)=\left(\frac{1}{2}, \frac{3}{2}\right)$.
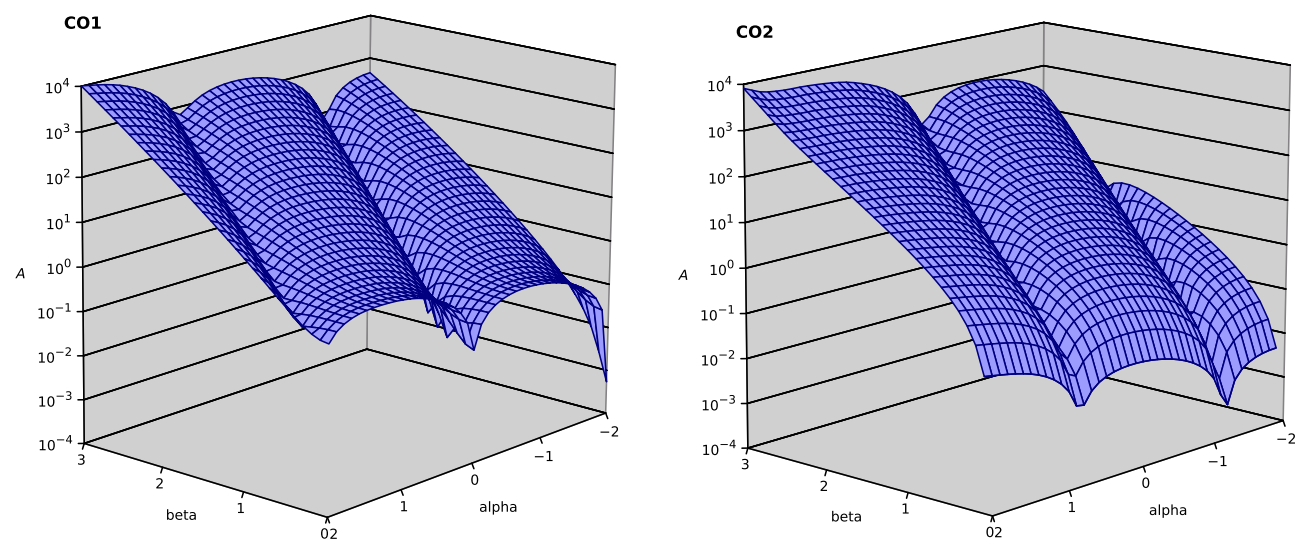

Fig. 12. Charge-controlled memristor when $\omega_{0}=1$ : locus of $A$ versus $(\alpha, \beta)$ for the $C O_{1}^{(\alpha, \beta)}$ and $C O_{2}^{(\alpha, \beta)}$.

\section{Conclusions}

This paper presented the fundamental aspects of fractional calculus, memristor systems and active device implementation of electrical impedances. Permeating those tools is also the notion of nonlinear dynamics. The generalization of derivatives and integrals encompasses both real and complex valued implementations. Therefore, a generalization of memristors is beyond real values and must address complex order objects. This paper initiated the debate and studied numerically several cases. The results are promising and stimulate further research on this vast topic either by exploring the fractional order or by analysing new types of nonlinearities.

\section{References}

[1] Kuhn TS. The structure of scientific revolutions. Chicago: Univ. of Chicago Press; 1962.

[2] Kurzweil R. The singularity is near: when humans transcend biology. London: Viking Press; 2005.

[3] Ross B. Fractional calculus. Math Mag 1977;50(3):115-22.

[4] Dugowson S. Les différentielles métaphysiques (histoire et philosophie de la généralisation de l'ordre de dérivation) Thèse, Université Paris Nord, Paris 1994.

[5] Josephs HJ. Oliver heaviside papers found at paignton in 1957. In: The institution of electrical engineers monograph No. 319, 1959. p. 70-6.

[6] Nahin PJ. Oliver heaviside: the life, work, and times of an electrical genius of the victorian age. Baltimore, London: The Johns Hopkins University Press; 2002.

[7] Mahon B. Oliver heaviside: maverick mastermind of electricity. London: Institution of Engineering and Technology; 2009.

[8] Fermi SUE, Pasta J. Studies of nonlinear problems. In: Segré E., editor. Published later in Collected Papers of Enrico Fermi, University of Chicago Press, Los Alamos report LA-1940, 1965. 
[9] Ford J. The fermi-pasta-ulam problem: paradox turns discovery. Phys. Rep. 1992;213(5):271-310.

[10] Weissert TP. The genesis of simulation in dynamics: pursuing the fermi-pasta-ulam problem. New York: Springer; 1997.

[11] Hu B, Campbell DK, Porter MA, Zabusky NJ. Fermi, pasta, ulam and the birth of experimental mathematics. Amer Sci 2009;97(6):214-21.

[12] Chua LO. Memristor - the missing circuit element. IEEE Trans Circ Theory 1971;18(2):507-19.

[13] Stewart Duncan R, Stanley Williams R, Strukov Dmitri B, Snider Gregory S. The missing memristor found. Nature 2008;97:80-3.

[14] Podlubny I. Fractional differential equations. An Introduction to Fractional Derivatives, Fractional Differential Equations, to Methods of Their Solution. Mathematics in Science and Engineering, vol. 198. San Diego: Academic Press; 1998.

[15] Srivastava HM, Kilbas AA, Trujillo JJ. Theory and applications of fractional differential equations. North-holland mathematics studies, vol. 204. Amsterdam: Elsevier; 2006.

[16] Oldham KB, Spanier J. The fractional calculus: theory and application of differentiation and integration to arbitrary order. New York: Academic Press; 1974.

[17] Miller KS, Ross B. An introduction to the fractional calculus and fractional differential equations. New York: John Wiley and Sons; 1993.

[18] Kilbas AA, Samko SG, Marichev OI. Fractional integrals and derivatives: theory and applications. Amsterdam: Gordon and Breach Science Publishers; 1993.

[19] Heaviside O. On operators in physical mathematics. Proc Roy Soc 1893;52:504-29.

[20] Gemant A. A method of analyzing experimental results obtained from elasto-viscous bodies. Physics 1936;7:311-7.

[21] Gemant A. On fractional differentials. Philos Mag 1938;25:540-9.

[22] Blair GWS. Analithical and integrative aspects of the stress-strain-time problem. J Sci Instrum 1944;21(5):80-4.

[23] Blair GWS. The role of psychophysics in rheology. J Colloid Sci 1947;2(1):21-32.

[24] Ross B. editor. Fractional Calculus and Its Applications. Proceedings of the International Conference, New Haven, Springer-Verlag, New York, 1974.

[25] Bruce West PG, Bologna Mauro. Physics of fractal operators. New York: Springer-Verlag; 2003.

[26] Magin RL. Fractional calculus in bioengineering. Redding, CT: Begell House Inc.; 2006.

[27] Fortuna L, Petras I, Caponetto Riccardo, Dongola Giovanni. Fractional order systems: modeling and control applications. Singapore: World Scientific 2010.

[28] Vinagre Blas M, Xue Dinguü, Feliu Vicente, Monje Concepción Alicia, Chen YangQuan. Fractional-order systems and controls. London: Springer; 2010.

[29] Mainardi Francesco, Tenreiro Machado J, Kiryakova Virginia. Recent history of fractional calculus. Commun Nonlinear Sci Numer Simul 2011;16(3):1140-53.

[30] Oustaloup A. Systèmes asservis linéaires d'ordre fractionnaire: théorie et pratique. Paris: Masson; 1983.

[31] Machado JAT. Analysis and design of fractional-order digital control systems. Syst Anal Model Simul 1997;27(2-3):107-22.

[32] Hilfer R. Applications of fractional calculus in physics. Singapore: World Scientific; 2000.

[33] Zaslavsky GM. Hamiltonian chaos and fractional dynamics. Oxford: Oxford University Press; 2005.

[34] Tenreiro Machado JA, Sabatier Jocelyn, Agrawal Om Prakash. Advances in fractional calculus: theoretical developments and applications in physics and engineering. Dordrecht, The Netherlands: Springer; 2007.

[35] Tarasov VE. Fractional Dynamics: Applications of Fractional Calculus to Dynamics of Particles, Fields and Media. Beijing, Heidelberg: Springer; 2010.

[36] Mainardi F. Fractional calculus and waves in linear viscoelasticity: an introduction to mathematical models. London: Imperial College Press; 2010.

[37] Tatom FB. The relationship between fractional calculus and fractals. Fractals 1995;3(1):217-29.

[38] Podlubny I. Geometric and physical interpretation of fractional integration and fractional differentiation. J Fract Calc Appl Anal 2002;5(4):367-86.

[39] Machado JAT. A probabilistic interpretation of the fractional-order differentiation. J Fract Calc Appl Anal 2003;6(1):73-80.

[40] Machado JAT. Fractional derivatives: probability interpretation and frequency response of rational approximations. Commun Nonlinear Sci Numer Simul 2009;14(9-10):3492-7.

[41] Saxena RK, Haubold HJ, Mathai AM. Mittag-leffler functions and their applications. J Appl Math 2011;2011:51. http://dx.doi.org/10.1155/2011/ 298628.

[42] Machado JAT. Fractional-order derivative approximations in discrete-time control systems. Syst Anal Model Simul 1999;34:419-34

[43] Podlubny I. Fractional-order systems and $\mathrm{PI}^{\lambda} \mathrm{D}^{\mu}$-controllers. IEEE Trans Automat Control 1999;44(1):208-13.

[44] Carlson GE, Halijak CA. Approximation of fractional capacitors $(1 / s)^{(1 / n)}$ by a regular Newton process. IEEE Trans Circ Theory 1964;CT-10(2):210-3.

[45] Roy SCD. On the realization of a constant-argument immitance of fractional operator. IEEE Trans Circ Theory 1967;14(3):264-374.

[46] Westerlund S, Ekstam L. Capacitor theory. IEEE Trans Dielect Electr Insul 1994;1(5):826-39.

[47] Westerlund S. Dead matter has memory. Causal consulting, Kalmar, Sweden, 2002.

[48] Bohannan GW. Analog realization of a fractional control element - revisited. In: Proc. of the 41st IEEE Int. Conf. on Decision and Control, Tutorial Workshop 2: Fractional Calculus Applications in Automatic Control and Robotics, Las Vegas, USA, 2002.

[49] Dutta PK, Biswas K, Sen S. Realization of a constant phase element and its performance study in a differentiator circuit. IEEE Trans Circ Syst II: Express Briefs 2006;53(9):802-6.

[50] Galhano Alexandra M, Boaventura Cunha J, Tenreiro Machado JA, Jesus Isabel S. Fractional calculus analysis of the electrical skin phenomena. In Agrawal OP, Sabatier Jocelyn, Tenreiro Machado J, editors. Advances in Fractional Calculus: Theoretical Developments and Applications in Physics and Engineering. Dordrecht, The Netherlands: Springer; 2007. p. 323-32.

[51] Krüger Klaus, Schafer I. Modelling of lossy coils using fractional derivatives. J Phys D: Appl Phys 2008;41(045001):1-8.

[52] Jesus IS, Machado JAT. Development of fractional order capacitors based on electrolyte processes. Nonlinear Dynam 2009;56(1-2):45-55.

[53] Galhano Alexandra M, Tenreiro Machado JA. Fractional order inductive phenomena based on the skin effect. Nonlinear Dynam. 2012;68(1-2):107-15.

[54] Qammar HK, Hartley TT, Lorenzo CF. Chaos in a fractional order chua's system. IEEE Trans Circ Syst I: Fund Theory Appl 1995;42(8):485-90.

[55] Podlubny Igor, Li Yan, Chen YangQuan. Stability of fractional-order nonlinear dynamic systems: Lyapunov direct method and generalized mittag-leffler stability. Comput Math Appl 2010;59(5):1810-21.

[56] Petráš I. Fractional-Order Nonlinear Systems: Modeling, Analysis and Simulation. Heidelberg: Springer; 2011.

[57] Machado JAT. Fractional dynamics of a system with particles subjected to impacts. Commun Nonlinear Sci Numer Simul 2011;16(12):4596-601.

[58] Luo Albert CJ, Baleanu Dumitru, Tenreiro Machado J. Fractional Dynamics and Control. New York: Springer; 2012.

[59] Kang Sung-Mo, Chua Leon O. Device modeling via basic nonlinear circuits elements. IEEE Trans Circ Syst 1980;27(11):1014-44.

[60] Auslander DM, Oster GF. The memristor: A new bond graph element. Trans ASME Dynam Syst Meas Control 1972;94(3):249-52.

[61] Kang Sung-Mo, Chua Leon O. Memristive devices and systems. Proc IEEE 1976;64(2):209-23.

[62] Chua LO. Nonlinear circuit foundations for nanodevices, Part I: the four-element torus. Proc IEEE 2003;91(11):1830-59.

[63] Chua Leon O, Di Ventra Massimiliano, Pershin Yuriy V. Circuits elements with memory: memristors, memcapacitors and meminductors. Proc IEEE 2009;97(10):1717-24.

[64] Williams RS. How we found the missing memristor. IEEE Spectrum 2008;45(12):28-35.

[65] Wang FY. Memristor for introductory physics. The City University of New York, Long Island, City, Aug. 4.

[66] Di Ventra Massimiliano, Pershin Yuriy V, La Fontaine Steven. Memristive model of amoeba learning. Phys Rev E 2009;80(2):80-3.

[67] Ebong I, Bhadviya Bhavitavya B, Mazumder P, Lu Wei, Jo Sung-Hyun, Chang Ting. Nanoscale memristor device as synapse in neuromorphic systems. Nano Lett 2010;10:1297-301.

[68] Chua LO, Di Ventra Massimiliano, Pershin Yuriy V. Circuits elements with memory: memristors, memcapacitors and meminductors. Proc IEEE 2009;97(10):1717-24.

[69] Doria-Cerezo Arnau, Jeltsema Dimitri. Memristive port-hamiltonian systems. Math Comput Model Dynam Syst 2010;16(2):75-93.

[70] Doria-Cerezo Arnau, Jeltsema Dimitri. Port-hamiltonian formulation of systems with memory. Proc IEEE 2012;100(6):1928-37. 
[71] Bruton LT. Network transfer functions using the concept of frequency dependent negative resistance. IEEE Trans Circ Theory 1969;16:406-8.

[72] Antoniou A. Novel RC-active-network synthesis using generalized immittance converters. IEEE Trans Circ Theory 1970;17(2):212-7.

[73] Smith KC, Sedra AS. A second generation current conveyor and its applications. IEEE Trans Circ Theory 1970;17(1):132-4.

[74] Soliman AM. Realization of frequency dependent negative resistance circuits using two capacitors and a single current conveyor. IEE Proc $1978 ; 125(12): 1336-7$

[75] Senani R. On the realization of floating active elements. IEEE Trans Circ Syst 1986;33(3):323-4.

[76] Saad Ramy A, Soliman Ahmed M. Two new families of floating FDNR circuits. J Elect Comput Eng 2010;2010 (Article ID 563761), 7 p.

[77] Williams AB. Electronic Filter Design Handbook. New York: McGraw-Hill; 1981.

[78] Valkenburg MEV. Analog Filter Design. New York: Holt, Rinehart and Winston; 1982.

[79] Ivo Petráš CC, YangQuan Chen, Fractional-order memristive systems. In: Proc. ETFA'09 Proceedings of the 14th IEEE International Conference on Emerging Technologies \& Factory Automation, Palma de Mallorca, Spain, 2009.

[80] Petráš I, Coopmans C, Chen YQ. Analogue fractional-order generalized memristive devices. In: Proc. of the ASME 2009 International Design Engineering Technical Conferences \& Computers and Information in Engineering Conference, San Diego, USA., 2009.

[81] Rekhviashvili SSh, Potapov AA. Memristor and the integral quantum hall effect. J Commun Tech Electron 2012;57(2):189-191. [Original Russian Text: Rekhviashvili SSh, Potapov AA. Radiotekhnika i Elektronika 2012;57(2):207-210].

[82] Lorenzo CF, Hartley TT, Adams JL. Complex-order distributions. In: Proc. of the ASME 2005 International Design Engineering Technical Conferences \& Computers and Information in Engineering Conference, Long Beach, CA., 2005.

[83] Adams JL, Hartley TT, Lorenzo CF. Conjugated-order differintegrals. In: Proc. of the ASME 2005 International Design Engineering Technical Conferences \& Computers and Information in Engineering Conference, Long Beach, CA., 2005.

[84] Barbosa Ramiro S, Silva Manuel F, Tenreiro Machado JA. Complex-order dynamics in hexapod locomotion. Signal Process 2006;86(10):2785-93.

[85] Silva Manuel F, Barbosa Ramiro S, Tenreiro Machado JA. Discretization of complex-order algorithms for control applications. J Vib Control 2008;14(910):1349-61.

[86] Tenreiro Machado JA, Pinto Carla MA. Complex order biped rhythms. Int J Bifur Chaos 2011;21(10):3053-61.

[87] Tenreiro Machado JA, Pinto Carla MA. Complex-order van der pol oscillator. Nonlinear Dynam 2011;65(3):247-54.

[88] Machado JAT, Pinto Carla MA. Complex order forced van der pol oscillator. J Vib Control, http://dx.doi.org/10.1177/1077546311429150.

[89] Machado JAT. Optimal controllers with complex order derivatives, J Opt Theory Appl, accept for publication. 